\title{
Mechanistic investigations on catalytic transfer
}

\section{hydrogenation of lignin derived monomers over $\mathrm{Ru}$}

\section{catalyst: Theoretical and kinetic studies}

\section{Tufeil Sartaj Khan, ${ }^{\dagger}$ Dheerendra Singht, Pragnya Paramita Samal,,,+}

Sailaja Krishnamurty,,,$\square$ Paresh Laxmikant. Dhepe ${ }^{*, t, \square}$

$\uparrow$ Catalysis and Inorganic Chemistry Division, CSIR-National Chemical Laboratory, Dr. Homi Bhabha Road, Pune 411008, India

\$ Physical and Materials Division, CSIR-National Chemical Laboratory, Dr. Homi Bhabha Road, Pune 411 008, India

$\square$ Academy of Scientific and Innovation Research (AcSIR), Gaziabad 201002, India

Contact: E-mail: pl.dhepe@ncl.res.in; Tel: +91 2025902024

Supporting information content

Total Number of pages $\quad: \quad 21$

Total number of figures $\quad: \quad 10$

Total number of tables $\quad: \quad 4$ 


\section{Characterization of catalyst}

1. XRD analysis: XRD spectra were recorded on Rigaku D MAX $\mathrm{Cu} \mathrm{K} \alpha$ radiation with $\lambda=1.54 \AA$ from 5 to $90^{\circ}$ at a scan rate of $4^{\circ} \cdot \mathrm{min}^{-1}$.

2. Nitrogen sorption studies: $\mathrm{N}_{2}$ physisorption experiments were done at $-196{ }^{\circ} \mathrm{C}$ using Autosorb iQ (Quantachrome Instruments, USA). Prior to analysis, samples were activated under vacuum at $200^{\circ} \mathrm{C}$ for $3 \mathrm{~h}$. The specific surface area was determined by BET method and pore size was calculated by BJH method.

3. CO chemisorption: $\mathrm{Ru}$ metal dispersion was determined through $\mathrm{CO}$ chemisorption using Quantachrome Autosorb- iQ instrument. Prior to sorption experiment, samples were insitu reduced in $\mathrm{H}_{2}$ at $150{ }^{\circ} \mathrm{C}$ for $2 \mathrm{~h}$. Subsequently, samples were evacuated for $2 \mathrm{~h}$ at same temperature and later were cooled to $50{ }^{\circ} \mathrm{C}$ under vacuum to record $\mathrm{CO}$ adsorption isotherm. The isotherm included both physisorption and chemisorption portion. Chemisorbed CO uptake was determined by extrapolating the linear portion of isotherm to zero pressure. The dispersion of Ru metal was calculated under assuming linear adsorption of $\mathrm{CO}$ on metal surface.

4. XPS analysis: The oxidation states of metals were analyzed by XPS (Perkin-Elmer PH 15000C) having X-ray source Al Ka radiation. The lens mode was LAXPS and energy step size was $0.1 \mathrm{eV}$. Spectrum peaks were deconvoluted via XPSPEAK-41. All binding energies were calibrated using the value of carbon $(\mathrm{C} 1 \mathrm{~S}=284.4 \mathrm{eV})$ as a reference.

5. TEM analysis: TEM images were obtained using FEI TECNAI T20 model instrument working at an accelerating voltage of $200 \mathrm{kV}$. Samples were dispersed in iso-propyl alcohol (IPA) via sonication and drop casted on carbon-coated copper grid.

6. ICP-OES analysis: ICP-OES analysis was done to know the metal contents in the catalysts by using SPECTRO ARCOS model FHS-12 (Germany). Before analysis, sample was digested in aquaregia. All ICP-OES samples were diluted with deionized water and filtered (through $0.22 \mu \mathrm{m}$ syringe filter). 
7. TPR analysis: The TPR studies were done by Micromeritics Autochem-2920 instrument in the temperature range of $50-800{ }^{\circ} \mathrm{C}$ in presence of $5 \% \mathrm{H}_{2}$ in $\mathrm{He}$ with a ramping rate of $5{ }^{\circ} \mathrm{C} \cdot \mathrm{min}^{-1}$. The catalyst was treated at $300{ }^{\circ} \mathrm{C}$ for $1 \mathrm{~h}$ in presence of He gas before the TPR analysis. The measurement of $\mathrm{H}_{2}$ consumption in the TPR analysis was done by a thermal conductivity detector (TCD) instrument.

8. $\mathrm{NH}_{3} / \mathrm{CO}_{2}$ TPD analysis: The acidity and basicity of the catalysts was investigated by temperature-programmed desorption of $\mathrm{NH}_{3}\left(\mathrm{NH}_{3}-\mathrm{TPD}\right)$ and $\mathrm{CO}_{2} \quad\left(\mathrm{CO}_{2}\right.$-TPD) using Micromeritics Autochem-2920 instrument. Prior to TPD run, the samples were activated at $400{ }^{\circ} \mathrm{C}$ in He flow $\left(40 \mathrm{~mL} \cdot \mathrm{min}^{-1}\right)$ for $1 \mathrm{~h}$. Subsequently, the temperature was brought down to $50{ }^{\circ} \mathrm{C}$ and $\mathrm{NH}_{3} / \mathrm{CO}_{2}$ was adsorbed by exposing the samples to a stream of $10 \% \mathrm{NH}_{3}$ in $\mathrm{He}$ $\left(30 \mathrm{~mL} \cdot \mathrm{min}^{-1}\right)$ and $10 \% \mathrm{CO}_{2}$ in $\mathrm{He}\left(30 \mathrm{~mL} \cdot \mathrm{min}^{-1}\right)$ for $0.5 \mathrm{~h}$. The temperature was then raised to $100{ }^{\circ} \mathrm{C}$ and flushed with $\mathrm{He}$ for $1 \mathrm{~h}$ at $100{ }^{\circ} \mathrm{C}$ to remove the physisorbed $\mathrm{NH}_{3} / \mathrm{CO}_{2}$. The desorption of $\mathrm{NH}_{3} / \mathrm{CO}_{2}$ was carried out from $100{ }^{\circ} \mathrm{C}$ to $800{ }^{\circ} \mathrm{C}$ 
Table S1. Summary on nitrogen sorption data.

\begin{tabular}{|l|c|c|c|}
\hline Catalyst & BET surface area & $\begin{array}{c}\text { Pore volume } \\
\left(\mathbf{\mathbf { m } ^ { 2 }} \cdot \mathbf{g}^{-\mathbf{1}}\right)\end{array}$ & $\begin{array}{c}\text { Pore radius } \\
\text { (nm) }\end{array}$ \\
\hline $\mathrm{Ru} / \mathrm{SiO}_{2}$ & & 0.51 & 3.35 \\
\hline $\mathrm{Al}_{2} \mathrm{O}_{3}-\mathrm{Acidic}$ & & 0.22 & 2.51 \\
\hline $\mathrm{Ru} / \mathrm{Al}_{2} \mathrm{O}_{3}$-Acidic & 305 & 0.22 & 2.59 \\
\hline $\mathrm{Ru} / \mathrm{Al}_{2} \mathrm{O}_{3}-\mathrm{Neutral}$ & 155 & 0.22 & 2.52 \\
\hline $\mathrm{Ru} / \mathrm{Al}_{2} \mathrm{O}_{3}-\mathrm{Basic}$ & 143 & 0.23 & 2.52 \\
\hline $\mathrm{SiO}-\mathrm{Al}_{2} \mathrm{O}_{3}$ & 147 & 0.48 & 3.01 \\
\hline $\mathrm{Ru} / \mathrm{SiO}_{2}-\mathrm{Al}_{2} \mathrm{O}_{3}$ & 374 & 0.43 & 2.29 \\
\hline
\end{tabular}


Table S2. Summary on ICP-OES analysis

\begin{tabular}{|l|c|c|}
\hline Catalyst & Theoretical $\mathbf{R u}$ wt\% & Experimental Ru wt\% \\
\hline $\mathrm{Ru} / \mathrm{SiO}_{2}$ & 0.50 & 0.42 \\
\hline $\mathrm{Ru} / \mathrm{Al}_{2} \mathrm{O}_{3}$-Acidic & 0.50 & 0.49 \\
\hline $\mathrm{Ru} / \mathrm{Al}_{2} \mathrm{O}_{3}$-Neutral & 0.50 & 0.45 \\
\hline $\mathrm{Ru} / \mathrm{Al}_{2} \mathrm{O}_{3}$-Basic & 0.50 & 0.49 \\
\hline $\mathrm{Ru} / \mathrm{SiO}_{2}-\mathrm{Al}_{2} \mathrm{O}_{3}$ & 0.50 & 0.49 \\
\hline
\end{tabular}


Table S3: CO chemisorption results for supported Ru catalysts

\begin{tabular}{|l|c|c|c|}
\hline Catalyst & $\begin{array}{c}\text { Average crystallite size } \\
(\mathbf{n m})\end{array}$ & $\begin{array}{c}\text { Metal } \\
\text { dispersion }(\%)\end{array}$ & $\begin{array}{c}\text { Monolayer } \\
\text { uptake }\left(\boldsymbol{\mu m o l} \bullet \mathbf{g}^{-1}\right)\end{array}$ \\
\hline $\mathrm{Ru} / \mathrm{SiO}_{2}$ & 11.8 & 11.4 & 5.6 \\
\hline $\mathrm{Ru} / \mathrm{Al}_{2} \mathrm{O}_{3}$-Acidic & 1.86 & 70 & 35 \\
\hline $\mathrm{Ru} / \mathrm{Al}_{2} \mathrm{O}_{3}$-Neutral & 2.18 & 61 & 30.3 \\
\hline $\mathrm{Ru} / \mathrm{Al}_{2} \mathrm{O}_{3}$-Basic & 2.27 & 59 & 29.1 \\
\hline $\mathrm{Ru} / \mathrm{SiO}_{2}-\mathrm{Al}_{2} \mathrm{O}_{3}$ & 3.05 & 44 & 21.6 \\
\hline
\end{tabular}


Table S4: $\mathrm{NH}_{3}$-TPD and $\mathrm{CO}_{2}$-TPD study of catalysts

\begin{tabular}{|c|c|}
\hline \multicolumn{2}{|c|}{$\mathrm{NH}_{3}$-TPD } \\
\hline Catalyst & Total acidity $\left(\mathrm{mmol}^{\prime} \mathrm{g}^{-1}\right)$ \\
\hline $\mathrm{Ru} / \mathrm{SiO}_{2}$ & - \\
\hline $\mathrm{Al}_{2} \mathrm{O}_{3}$-Acidic & 0.23 \\
\hline $\mathrm{Ru} / \mathrm{Al}_{2} \mathrm{O}_{3}$-Acidic & 0.46 \\
\hline $\mathrm{Ru} / \mathrm{Al}_{2} \mathrm{O}_{3}$-Neutral & 0.16 \\
\hline $\mathrm{SiO}_{2}-\mathrm{Al}_{2} \mathrm{O}_{3}$ & 0.54 \\
\hline $\mathrm{Ru} / \mathrm{SiO}_{2}-\mathrm{Al}_{2} \mathrm{O}_{3}$ & 0.76 \\
\hline
\end{tabular}

\begin{tabular}{|l|c|}
\hline \multicolumn{2}{|c|}{ COO$_{2}$-TPD } \\
\hline Catalyst & Total basicity (mmol•g-1) \\
& \\
\hline $\mathrm{Ru} / \mathrm{Al}_{2} \mathrm{O}_{3}$-Basic & 0.17 \\
\hline
\end{tabular}




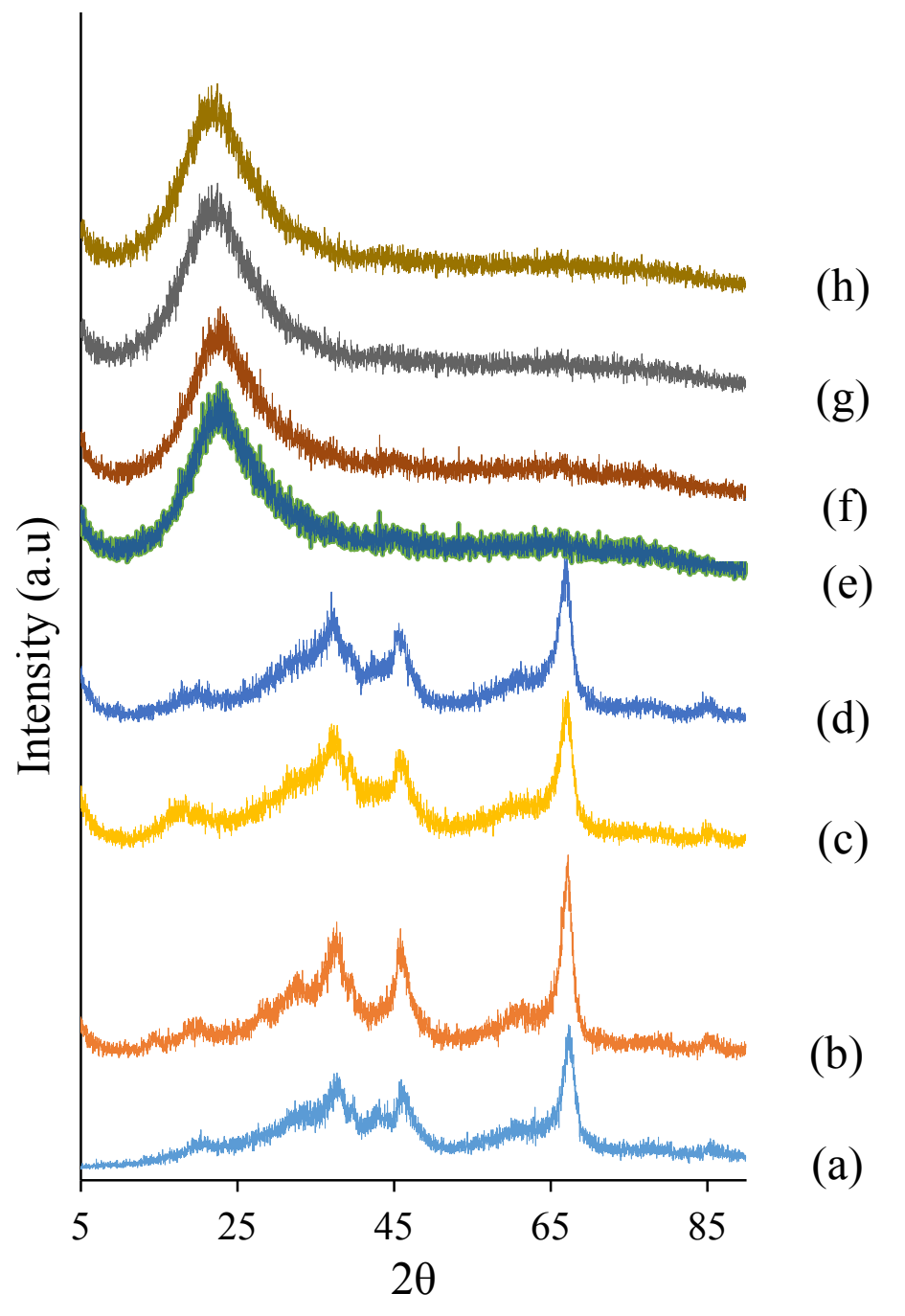

Figure S1. XRD patterns of support and supported $\mathrm{Ru}$ catalysts. (a) $\mathrm{Al}_{2} \mathrm{O}_{3}$, (b) $\mathrm{Ru} / \mathrm{Al}_{2} \mathrm{O}_{3^{-}}$ Acidic (c) $\mathrm{Ru} / \mathrm{Al}_{2} \mathrm{O}_{3}$-Neutral, (d) $\mathrm{Ru} / \mathrm{Al}_{2} \mathrm{O}_{3}$-Basic, (e) $\mathrm{SiO}_{2}-\mathrm{Al}_{2} \mathrm{O}_{3}$, (f) $\mathrm{Ru} / \mathrm{SiO}_{2}-\mathrm{Al}_{2} \mathrm{O}_{3}$, (g) $\mathrm{SiO}_{2}$, and (h) $\mathrm{Ru} / \mathrm{SiO}_{2}$. 

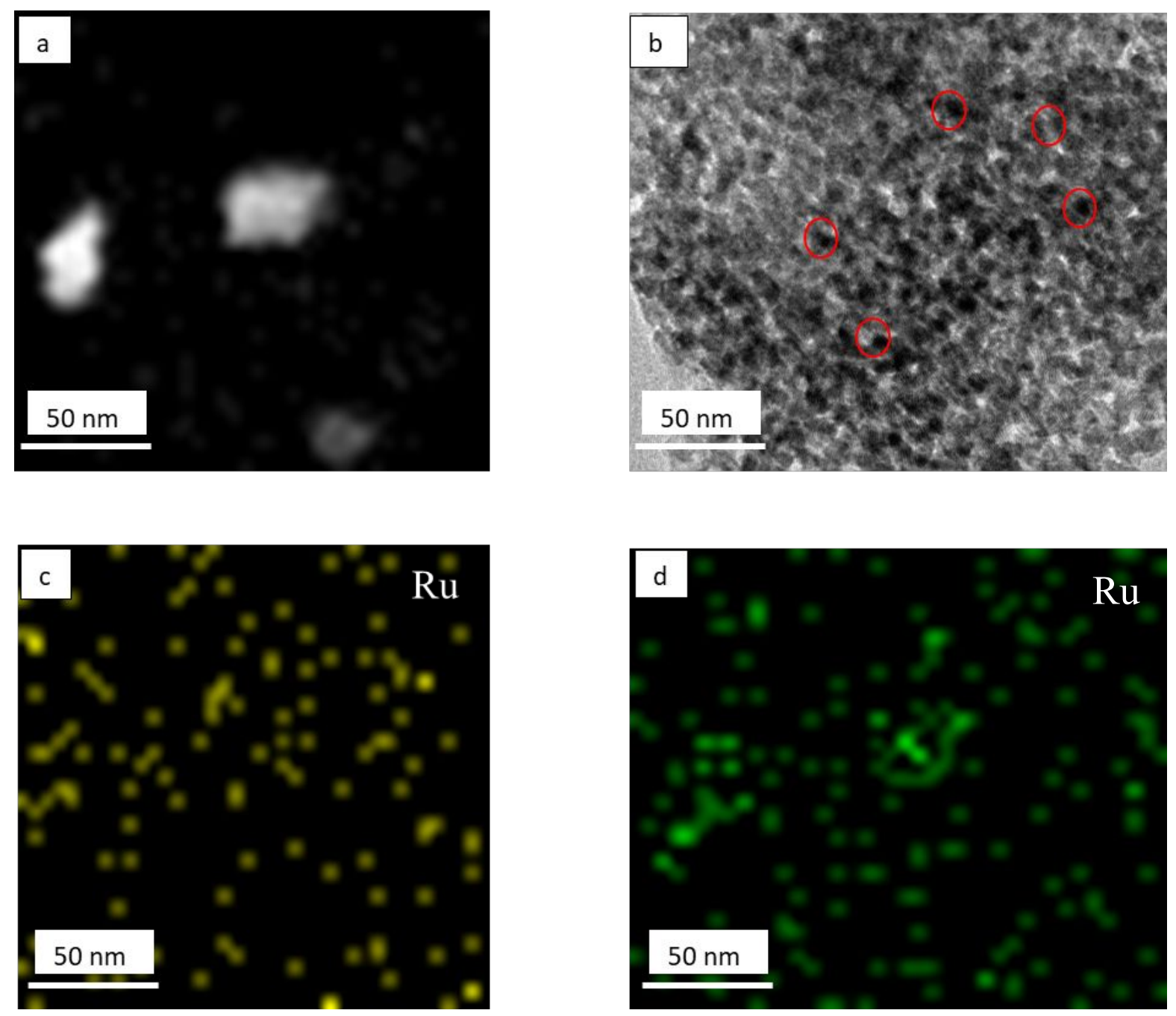

Figure S2a. (a) HAADF-STEM, (b) TEM, (c, d) EDX Elemental mapping of $\mathrm{Ru} / \mathrm{Al}_{2} \mathrm{O}_{3}$ Acidic. 


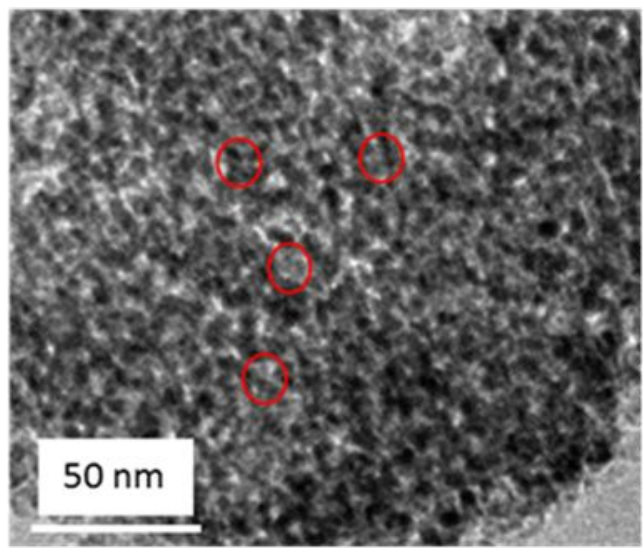

(a)

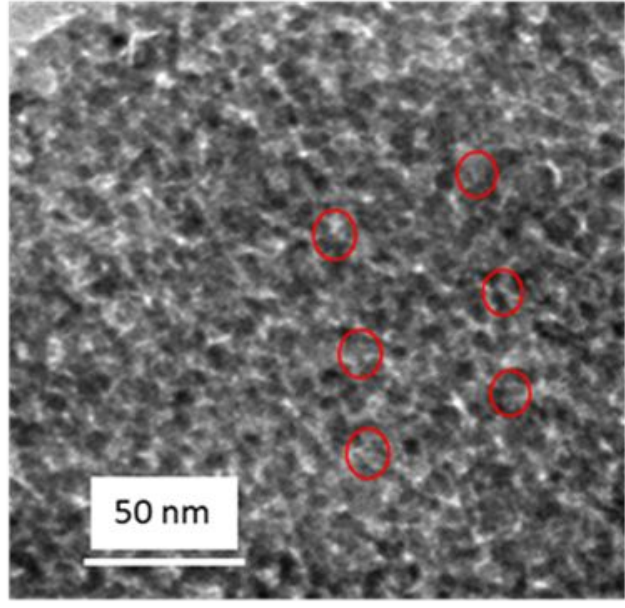

(b)

Figure S2b. TEM image of (a) $\mathrm{Ru} / \mathrm{Al}_{2} \mathrm{O}_{3}-\mathrm{Neutral}$ (b) $\mathrm{Ru} / \mathrm{Al}_{2} \mathrm{O}_{3}-$ Basic. 


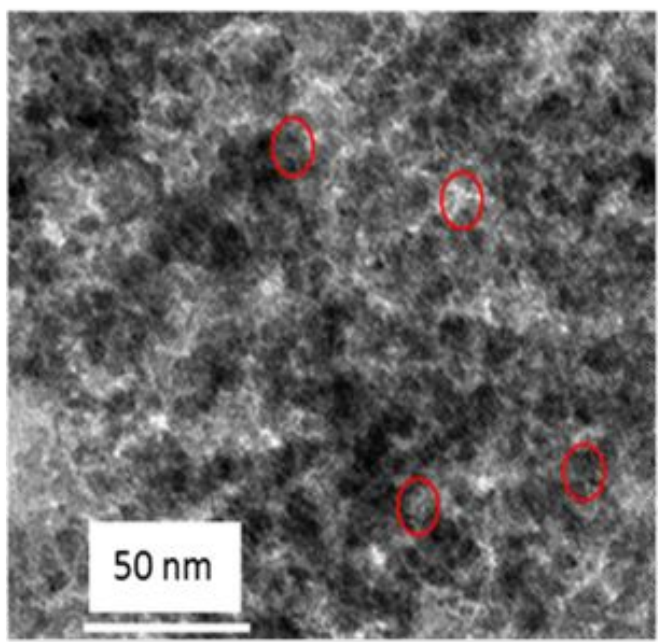

(a)

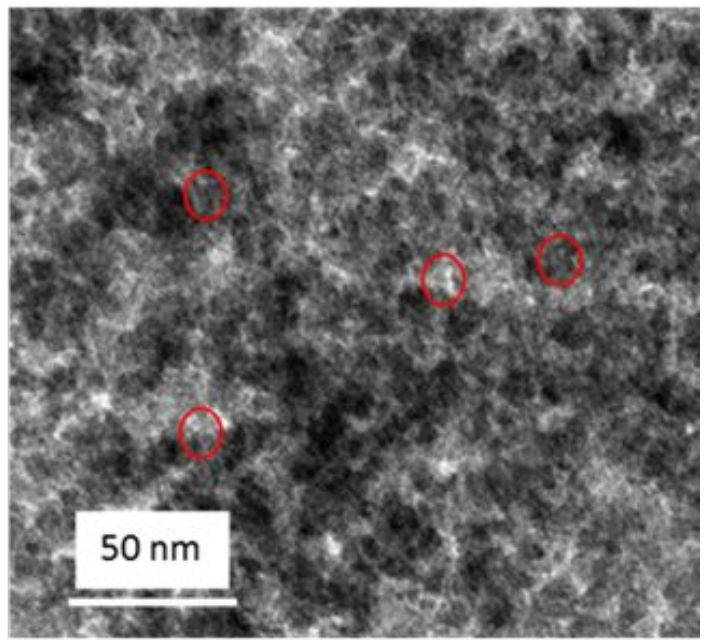

(b)

Figure S3. TEM image of (a) $\mathrm{Ru} / \mathrm{SiO}_{2}$, (b) $\mathrm{Ru} / \mathrm{SiO}_{2}-\mathrm{Al}_{2} \mathrm{O}_{3}$ 


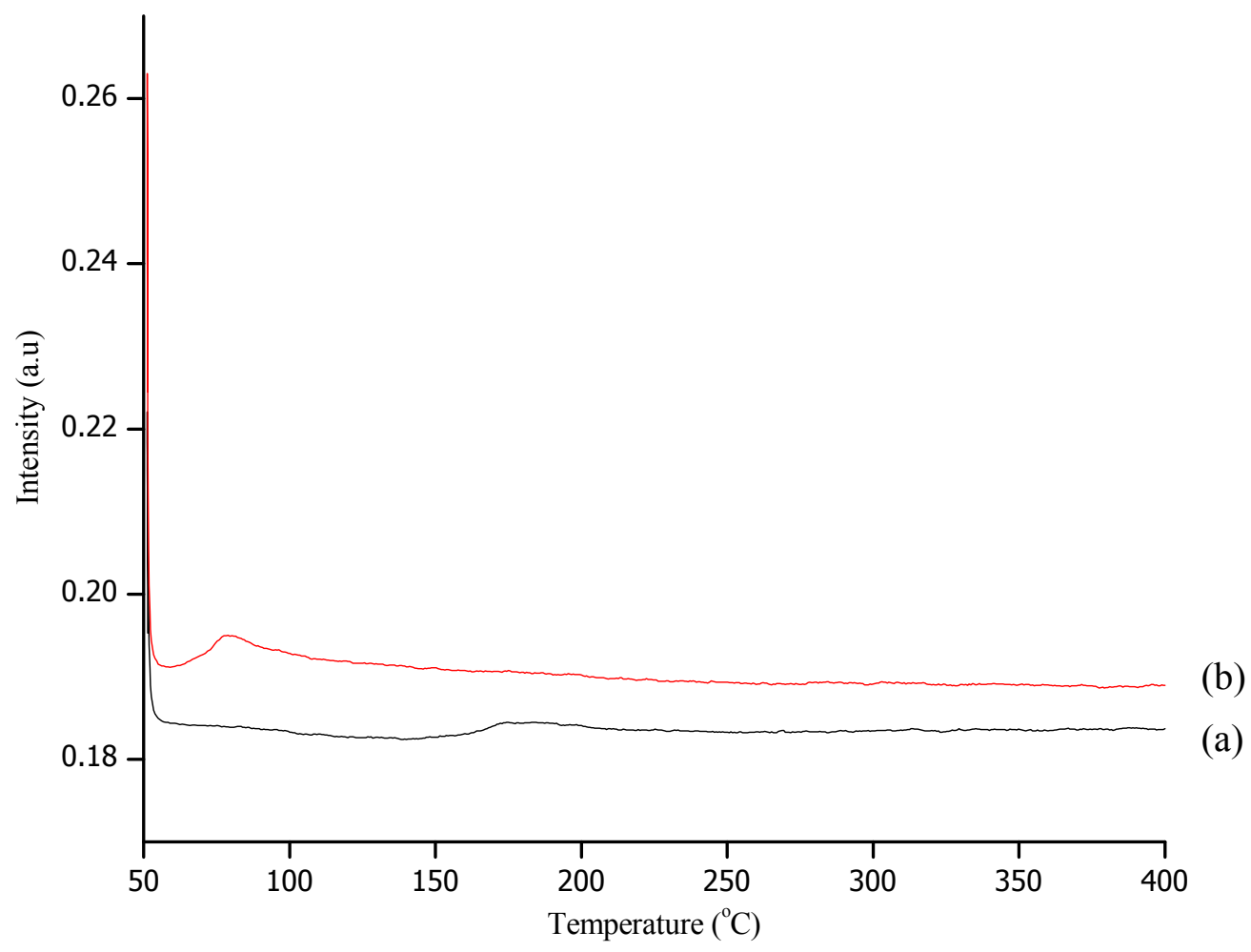

Figure S4: TPR of support and supported Ru metal catalyst (a) $\mathrm{Al}_{2} \mathrm{O}_{3}$, (b) $\mathrm{Ru} / \mathrm{Al}_{2} \mathrm{O}_{3}$-Acidic. 
$\mathrm{C} 1 \mathrm{~s}$ and $\mathrm{Ru} 3 \mathrm{~d} 3 / 2$

B. $\mathrm{E} .=284.3 \mathrm{eV}, \mathrm{Ru} / \mathrm{RuOx}$

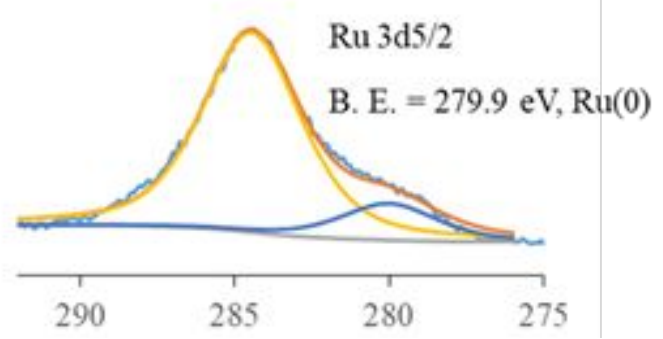

B.E. $(\mathrm{eV})$
Ru 3p5/2, B. E. $=461.2 \mathrm{Ru}(0)$

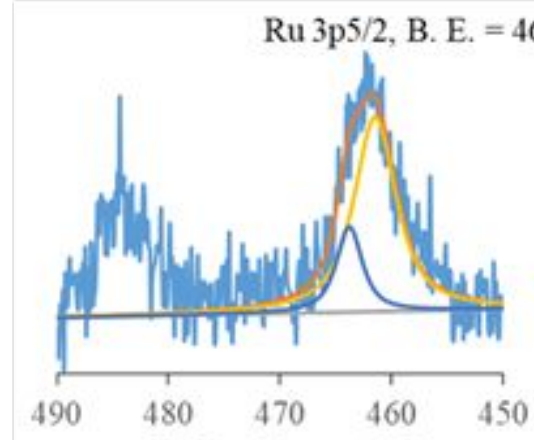

B.E. $(\mathrm{eV})$

Figure S5a: Deconvoluted XPS spectrum of $\mathrm{Ru} / \mathrm{Al}_{2} \mathrm{O}_{3}$-Acidic catalyst.
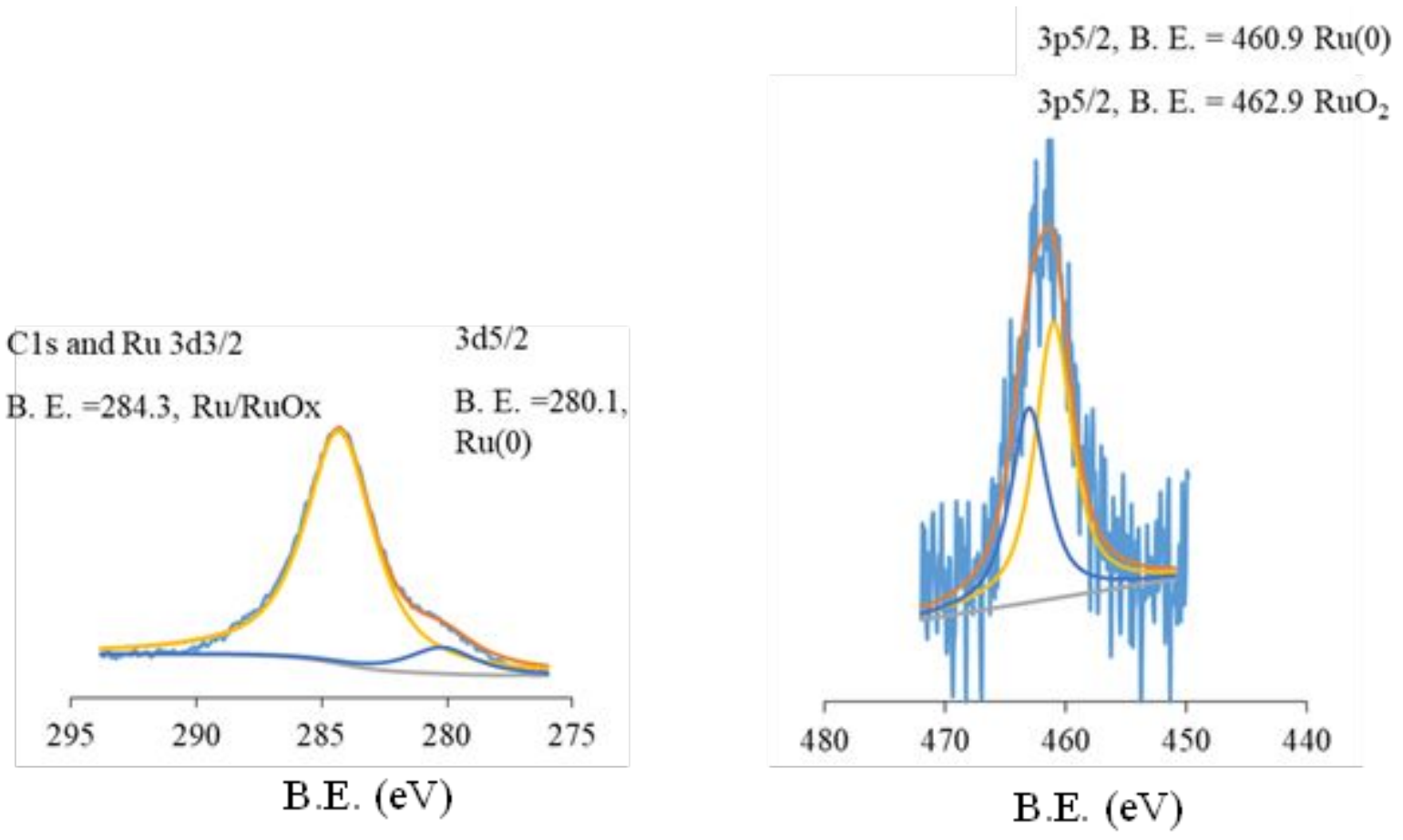

Figure S5b: Deconvoluted XPS spectrum of $\mathrm{Ru} / \mathrm{Al}_{2} \mathrm{O}_{3}$-Basic catalyst. 
$\mathrm{C} 1 \mathrm{~s}$ and $\mathrm{Ru} 3 \mathrm{~d} 3 / 2$

B. E. $=284.3, \mathrm{Ru} / \mathrm{RuOx}$

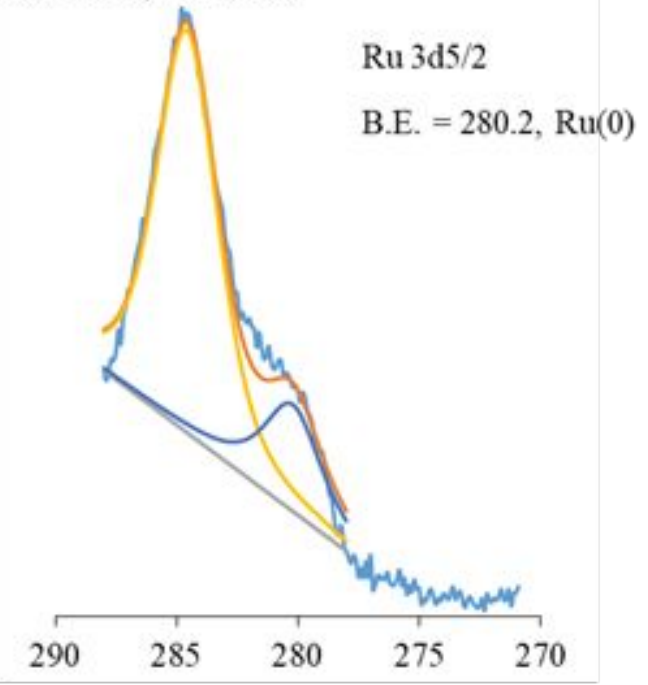

B.E. $(\mathrm{eV})$

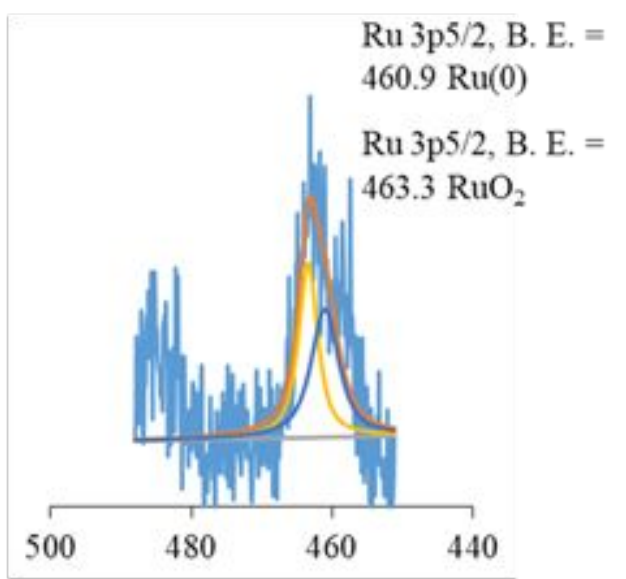

B.E. $(\mathrm{eV})$

Figure S5c: Deconvoluted XPS spectrum of $\mathrm{Ru} / \mathrm{Al}_{2} \mathrm{O}_{3}-\mathrm{Neutral}$ catalyst.
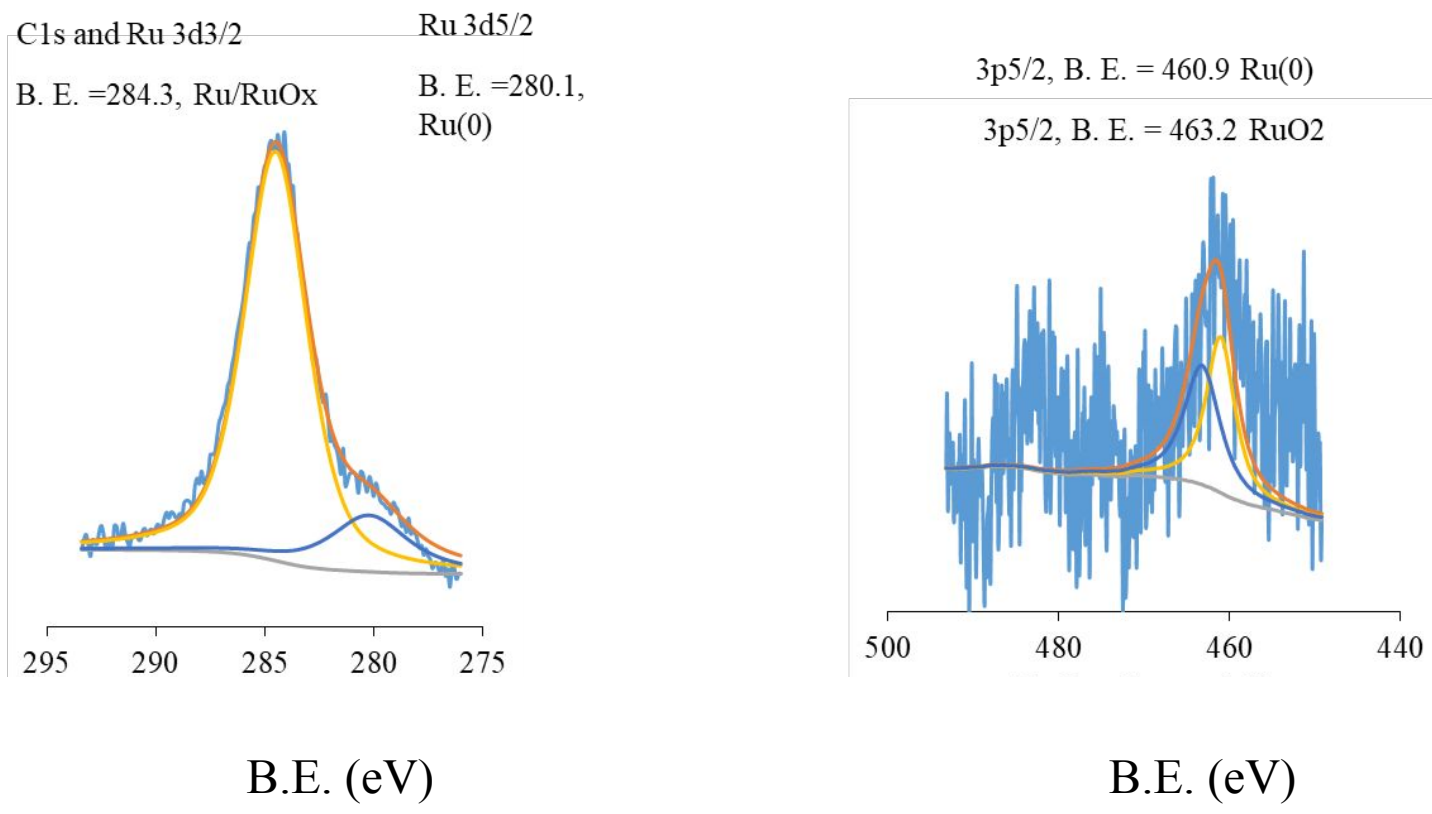

Figure S5d: Deconvoluted XPS spectrum of $\mathrm{Ru} / \mathrm{SiO}_{2}-\mathrm{Al}_{2} \mathrm{O}_{3}$ catalyst. 


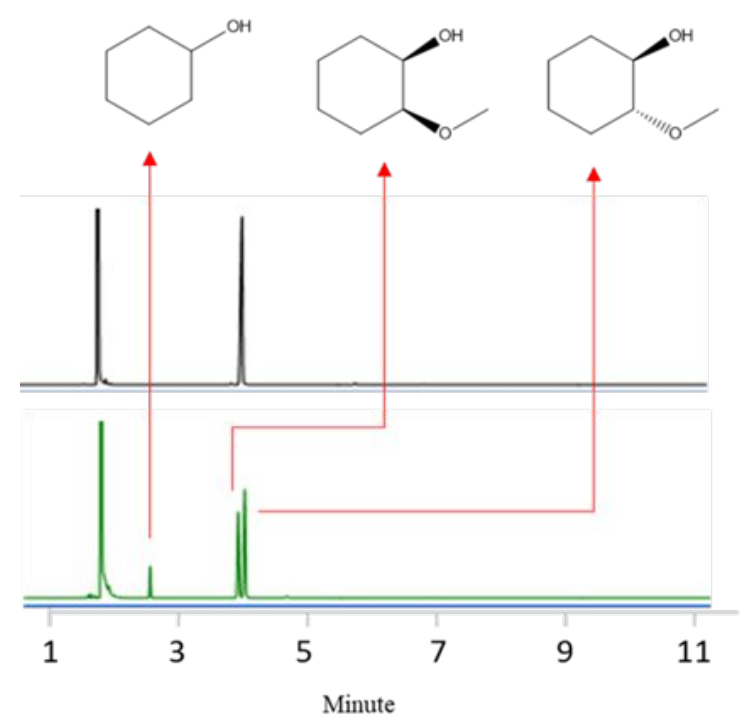

(b)

(a)

(A)

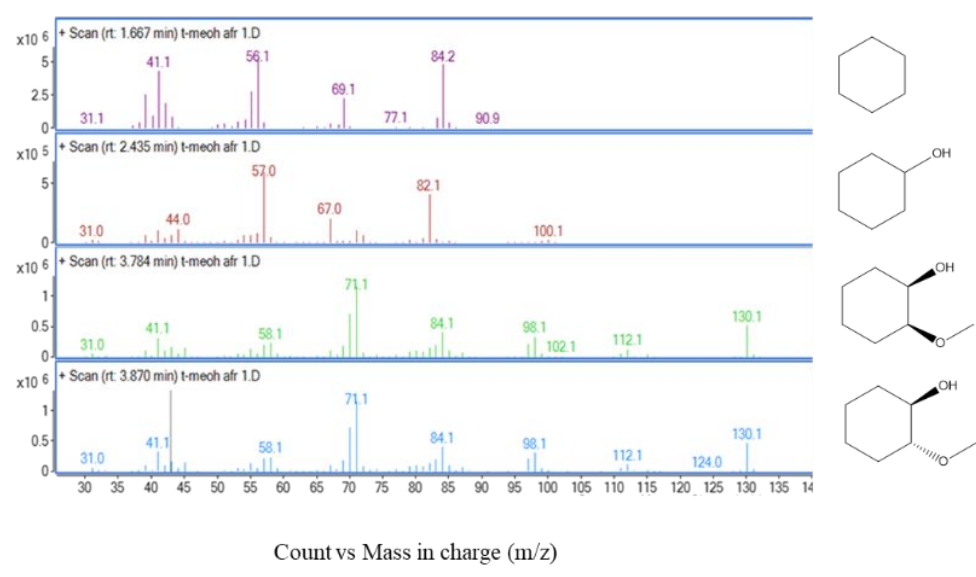

(B)

Figure S6. Reaction with (1S,2S)-2-methoxycyclohexanol (A) GC-MS profile for (a) after reaction (b) before reaction (B) GC-MS fragmentation profile.

Reaction condition: $225^{\circ} \mathrm{C}, \mathrm{H}_{2}, 1 \mathrm{MPa} ; 1 \mathrm{~h}$. 


\section{DFT details}

$\mathrm{Ru}(0001)$ plane is modelled as a periodic slab and optimized using Density Functional Theory as implemented in Vienna $\mathrm{Ab}$ initio Simulation Package (VASP) ${ }^{1}$ software with PBE functional ${ }^{2}$. Projected augmented wave (PAW) ${ }^{3}$ method is employed with an energy cut-off of $400 \mathrm{eV}$ to describe plane wave basis set. An energy threshold of $10^{-7} \mathrm{eV}$ and a force threshold of $10^{-5} \mathrm{eV} / \mathrm{A}$ were used as the optimization criteria. To avoid the interactions between the neighbouring layers of the surfaces, a vacuum space of $20 \AA$ is created along the Z-direction. The structural optimization of all geometries is carried out using the conjugate gradient method. For geometries optimization, the Monkhorst-Pack generated $(4 \times 4 \times 1)$ set of k-point grid is used. Guaiacol, (1R,2S)-2-methoxycyclohexanol, (1S,2S)-2-methoxycyclohexanol, and Cyclohexanol molecules are adsorbed on to the $\mathrm{Ru}(0001)$ surface and the complexes optimized based on the experimental pathway of conversion Guaiacol to Cyclohexanol in presence of $\mathrm{Ru}$ as an active catalyst. Existence of no negative stretching frequencies indicates the stability intermediates on $\mathrm{Ru}(0001)$ surface.

The adsorption energies of the molecules on the $\mathrm{Ru}$ surface are calculated as $\mathrm{E}_{\mathrm{ad}}=\mathrm{E}($ molecule-Ru $)-[\mathrm{E}($ molecule $)+\mathrm{E}(\mathrm{Ru})]$

Where $\mathrm{E}($ molecule- $\mathrm{Ru})$ represents the energy of the molecule adsorbed on $\mathrm{Ru}, \mathrm{E}$ (molecule) represents the energy of the molecule and $\mathrm{E}(\mathrm{Ru})$ represents the energy of the Ru surface.

The electronic properties of the three complexes are analyzed using Density of states (DOS) and Charge density difference (CDD) plots. 


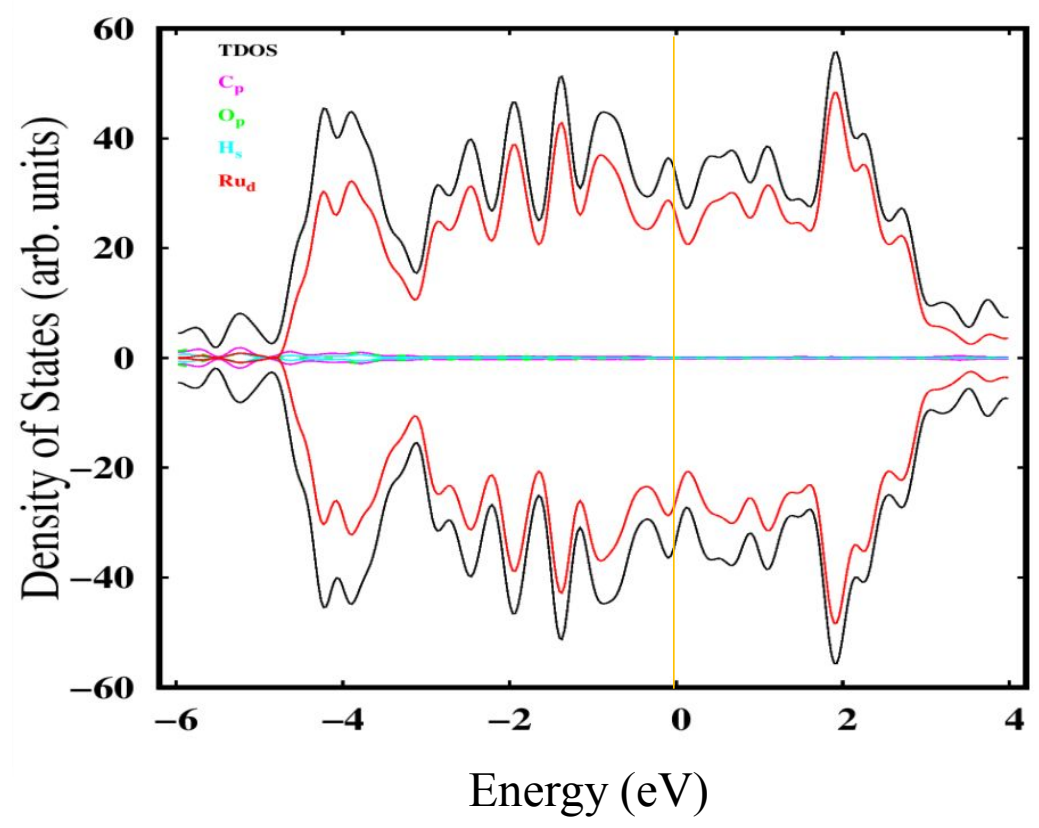

Figure S7. Density of states (DOS) plot for (1S,2S)-2-methoxycyclohexanol intermediate adsorbed on $\mathrm{Ru}$ (0001). 


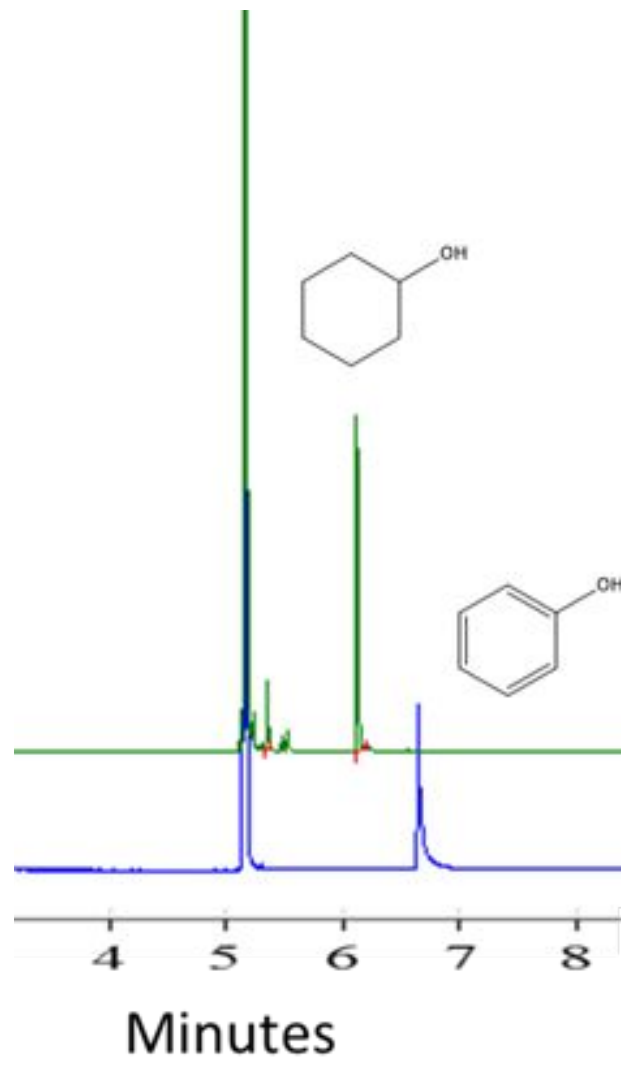

(b)

(a)

Figure S8: Representative GC profile. GC profile. Phenol/surface exposed Ru metal molar ratio, 35520; 2.13 wt\% Phenol in IPA; $0.7 \mathrm{MPa} \mathrm{N}{ }_{2} ; 225^{\circ} \mathrm{C} ; 1000 \mathrm{rpm}$. 


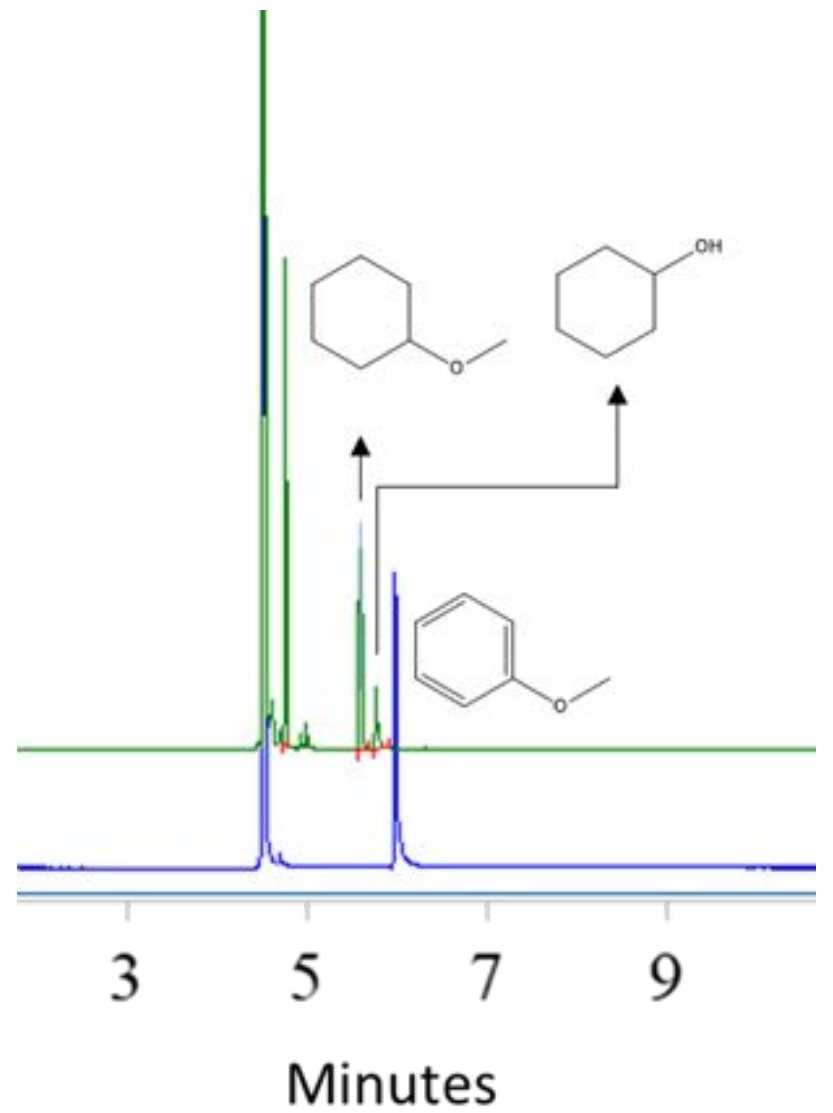

(b)

(a)

Figure S9. Representative GC profile. GC profile. Anisole/surface exposed Ru metal molar ratio, 35520; $2.13 \mathrm{wt} \%$ Anisole in IPA; $0.7 \mathrm{MPa} \mathrm{N} \mathrm{N}_{2} ; 225^{\circ} \mathrm{C} ; 1000 \mathrm{rpm}$. 


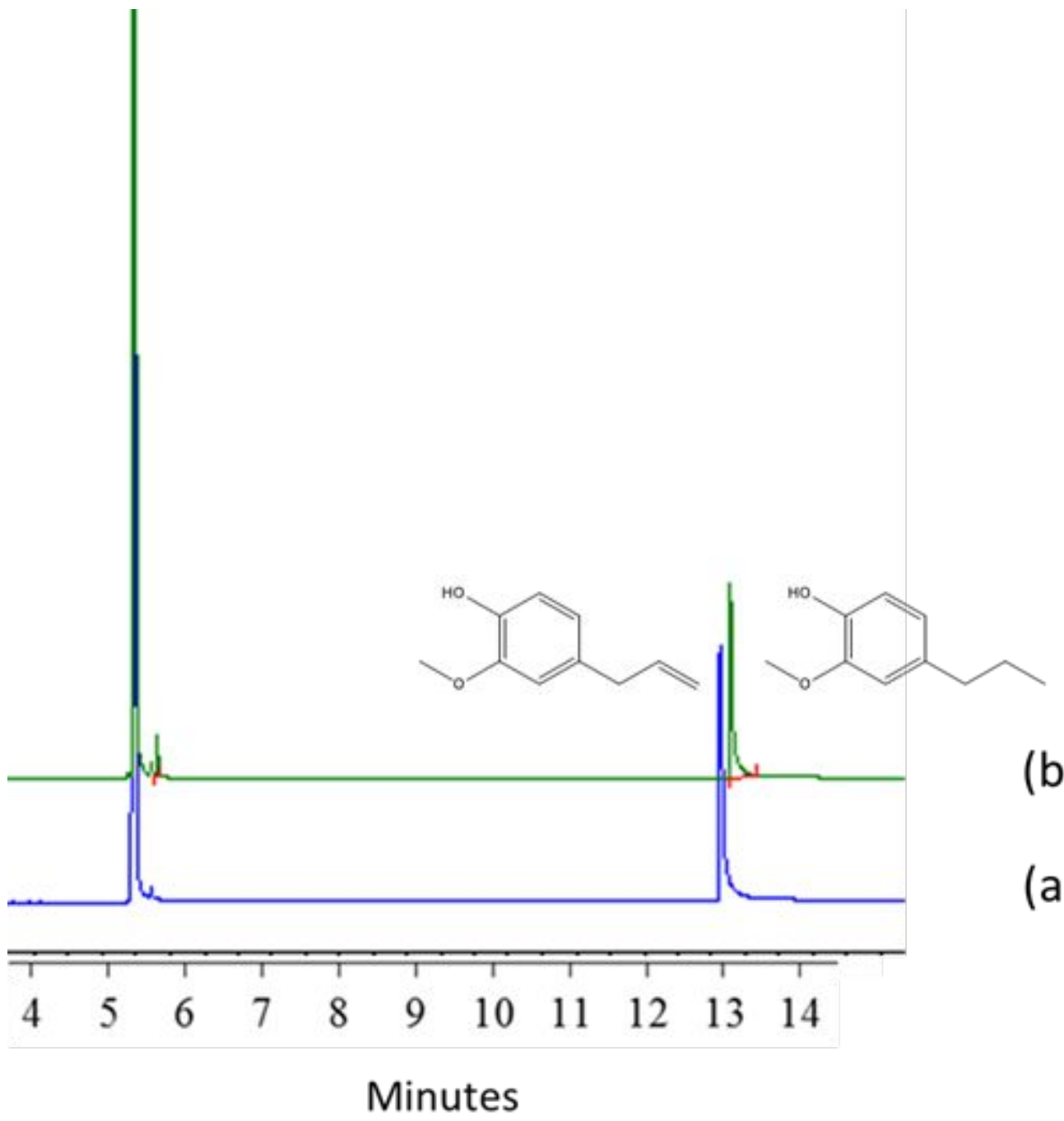

Figure S10: Representative GC profile. GC profile. Eugenol/surface exposed Ru metal molar ratio, 35520; $2.13 \mathrm{wt} \%$ Eugenol in IPA; $0.7 \mathrm{MPa} \mathrm{N}_{2} ; 225^{\circ} \mathrm{C} ; 1000 \mathrm{rpm}$. 


\section{Reference:}

1. Kresse, G.; Furthmüller, J.; Efficiency of ab-initio total energy calculations for metals and semiconductors using a plane-wave basis set. Comput. Mater. Sci. 1996, 6 (1), 15-50. DOI: 10.1016/0927-0256(96)00008-0

2. $\quad$ Perdew, J. P.; Burke, K.; Ernzerhof, M.; Generalized Gradient Approximation Made Simple. Phys. Rev. Lett. 1996, 77 (18), 3865-3868. DOI: 10.1103/PhysRevLett.77.3865

3. Blöchl, P. E.; Projector augmented-wave method. Phys. Rev. B. 1994, 50 (24), 17953 17979. DOI: 10.1103/PhysRevB.50.17953 